\title{
Arterio-venous fistula with pseudoaneurysm of renal artery
}

\author{
Breza J Jr, Javorka V Jr, Mizickova M, Goncalves FM, Breza J Sr \\ Department of Urology and Radiology, University Hospital Bratislava, Slovakia.
}

\begin{abstract}
Introduction: The authors describe a rare but potentially severe, sometimes even life-threatening complication occurring after various invasive renal interventions. The cause that gives rise to a fistula formation between close branches of renal artery and vein is an injury of these vessels during resection of a localised renal tumour, renal biopsy, percutaneous nephrostomy, and even blunt injury of the kidney. Blood flows under pressure from renal artery, pushes the surrounding renal parenchyma open, and creates a cavity varying in size and shape. This pseudoaneurysm may communicate with the collecting system of the kidney, in which case it may lead to haematuria, one of the typical symptoms of arteriovenous fistula.

Material and methods: The authors observed this complication in 8 patients ( 6 males aged from 56 to 70 , average 61.7, and two females aged 28 and 54). In 7 patients, the fistula developed after resection of a localised renal tumour, in one patient after percutaneous nephrolitholapaxy of a stone in the kidney. The fistulae manifested themselves between 7 an 21 days after surgery by massive haematuria in 4 patients and by intermittent macroscopic haematuria in 3 patients. In one patient the fistula was asymptomatic while the pseudoaneurysm at the place of pathological connection of renal veins was found during ultrasonographic examination after renal resection. Results: In all patients, the diagnosis of arteriovenous fistula and pseudoaneurysm of renal artery was achieved by ultrasonographic and/or CT examinations. The highest diagnostic value can be ascribed to digital subtraction renoangiography by Seldinger method that subsequently allowed active treatment by introducing an endovascular coil. By this method we cured 5 fistulae. In two patients, urgent nephrectomies were needed because of massive life-threatening haematuria. One of arteriovenous fistulae underwent spontaneous thrombosis.

Conclusion: The symptomatic arteriovenous fistula requires fast and precise diagnosis subsequently enabling efficient treatment. Endovascular manipulation is a method allowing the preservation of kidney affected by arteriovenous fistula with renal artery pseudoaneurysm (Fig. 7, Ref. 21). Full Text in PDF www.elis.sk.

Key words: arteriovenous fistula, renal artery, pseudoaneurysm, fistula, haematuria, nephrostomy, percutaneous nephrostomy.
\end{abstract}

Creation of an arteriovenous fistula with a subsequent development of pseudoaneurysm at the site of pathological connection of the injured branches of renal artery and vein is a rare specific complication that may occur after various invasive renal interventions. It is most frequently caused by injury of vessels during extirpation of a localised tumour of the kidney or when resecting a part of the kidney affected by tumour. On reconstructing the kidney by suturing the two opposite walls of the cavity after removing the tumour and bringing the two resection surfaces into contact, the injured vessels may get into mutual communication. Blood flows out through the hole in the artery under pressure into the space between the two resection walls, which gives rise to a void referred to as pseudoaneurysm. The pseudoaneurysm increases in size, pushes the surrounding parenchyma open and may become symptomatic (Baláž et al, 2007).

This complication may arise also after other invasive diagnostic or therapeutic interventions such as renal biopsy (Kaufman et al, 1969), percutaneous operations on the kidney or seldom also

$\overline{\text { Department of Urology and Radiology, University Hospital Bratislava, }}$ Slovakia.

Address for correspondence: J. Breza Jr, MD, Department of Urology and Radiology, University Hospital Bratislava, Limbova 5, SK-833 05 Bratislava, Slovakia. after a blunt injury of the kidney (Gainza et al, 1995, Swana et al, 1996, Ibanez Pradas et al, 2006). The arteriovenous fistula may be part of complex pathological changes in the tumour tissue of hypervascularised adenocarcinoma of the kidney (Sell et al, 1986).

The arteriovenous fistula exceptionally occurs at the place of stump of renal vessels after nephrectomy, namely after en masse ligation of renal artery and vein (Zutombel, 1973).

The objective of the work is to present our own experience with this scarce but potentially severe or even life-threatening complication occurring after various invasive renal interventions.

\section{Material and methods}

The authors have observed arteriovenous fistulae with pseudoaneurysms in 8 patients: 6 males aged 56 to 70 (average 61.7, median 60.5, standard deviation 4.6) and 2 females aged 28 and 54. In 7 patients, the fistula developed after extirpation of a localised tumour, in one man after percutaneous removal of a urinary stone from the kidney. The fistulae manifested themselves by massive haematuria in 4 patients and intermittent macroscopic haematuria in 3 patients on day 12.1 on the average ( 7 to 21 , median 11.0 , standard deviation 4.5) after operation. In none of the patients, the fistula showed itself by bleeding from the drain tube used for 


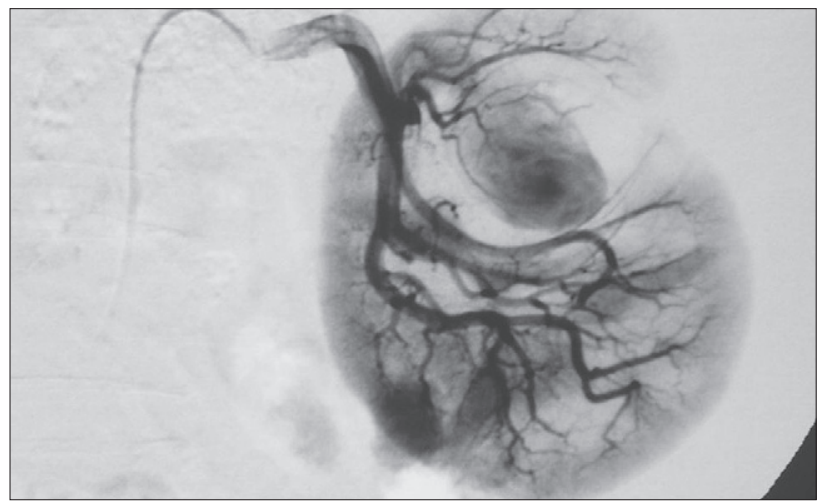

Fig. 1. Renoangiography: Arterio-venous fistula with one pseudoaneurysm of renal artery.

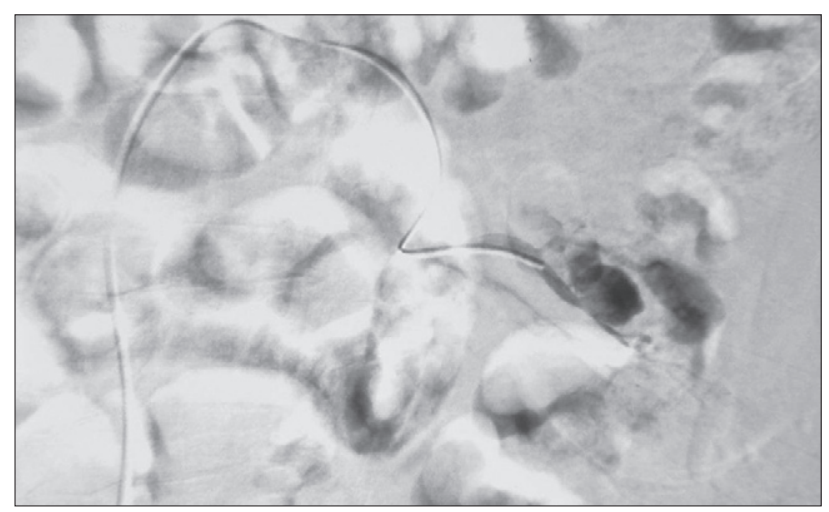

Fig. 2. Renoangiography: Arterio-venous fistula with two pseudoaneurysms of renal artery.

draining the perirenal space after the operation. In one patient, the fistula was asymptomatic and the pseudoaneurysm at the place of pathological connection of renal vessels was found by standard ultrasonographic check up after kidney resection.

\section{Results}

The operations leading to the development of arteriovenous fistulae were carried out with no complications. Similarly, the im- mediate postoperative course was appropriate. In all 7 patients, the localised renal tumours were removed in toto with negative surgical margins. Histological examination confirmed clear cell renal carcinomas in 5 patients, papillary renal carcinoma in one patient, and a complex cyst Bosniak III with a tiny focus of adenocarcinoma in the wall of the cyst in one patient. In the case of nephrolithiasis, the stone was solitary, $2 \mathrm{~cm}$ large in diameter. It was disintegrated mechanically in the renal pelvis and all fragments were removed. After the operation in this patient, temporary urine diversion was ensured by nephrostomy.

In each of six patients, one pseudoaneurysm developed (Fig. 1), in one patient there were two pseudoaneurysms (Fig. 2), and in one patient we diagnosed one multilobular pseudoaneurysm (Figs $3 \mathrm{a}-\mathrm{c}$ and 4). The voids were from 1 to $4 \mathrm{~cm}$ in diameter.

In all patients, the diagnosis of arteriovenous fistula and pseudoaneurysm of the renal artery was based on ultrasonographic and CT examinations. In sonographic examinations, hypoechogenous to anechogenous formations can be seen in the parenchyma of the affected kidney, afterwards Doppler flowmetry proved a typical turbulent blood flow in the fistula or in the void of the pseudoaneurysm (Fig. 5). The highest diagnostic value belongs to digital subtraction renoangiography by Seldinger's method that subsequently enabled active treatment by applying coil embolisation. Local thrombosis develops around the coils and the arteriovenous fistula occludes. By this method we cured 5 fistulae (Figs 6 and 7). In all patients, their clinical state improved immediately after intervention, haematuria was stopped, laboratory parameters were normalised. We did not observe hypertension as a consequence of ischaemia of the renal parenchyma induced by occlusion of the arteriovenous fistula. In two patients, urgent nephrectomy was inevitable because of life-threatening massive haematuria with incipient haemorrhagic shock. The postoperative course in the two patients, one man and one woman, was without complications. One arteriovenous fistula of renal artery healed spontaneously.

\section{Discussion}

The formation of arteriovenous fistula is a rare complication occurring after various diagnostic and therapeutic renal interventions. Arteriovenous fistulae of renal artery may also be a demon-

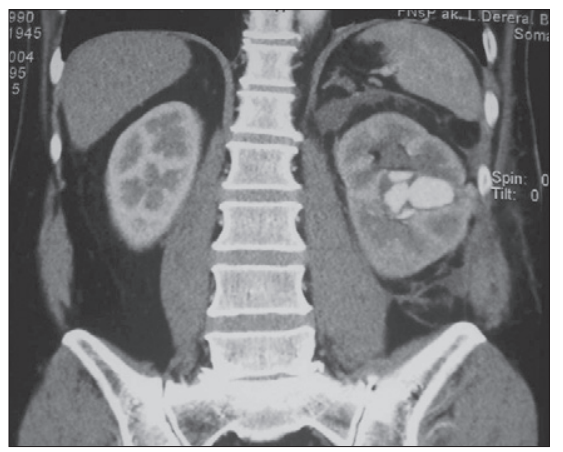

Fig. 3 a. CT - coronary scan: Arterio-venous fistula with multilobular pseudoaneurysm of renal artery.

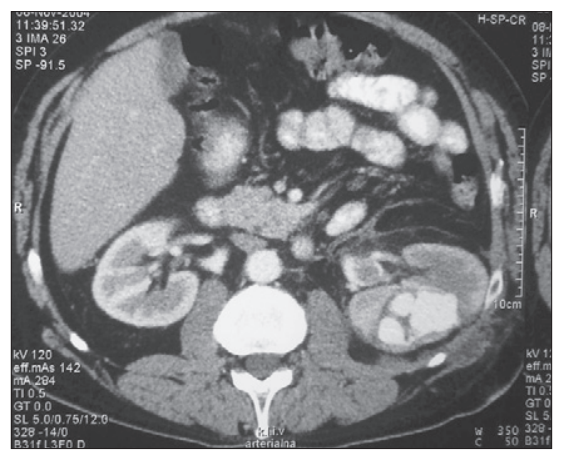

Fig. 3 b. CT - transverse scan: Arterio-venous fistula with
renal artery.

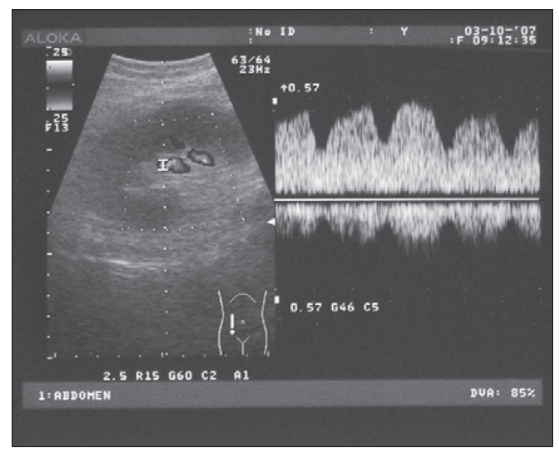

Fig. 3 c. Doppler ultrasonic flowmetry demonstrating typical turbulent blood flow in renal artery pseudoaneurysm. 


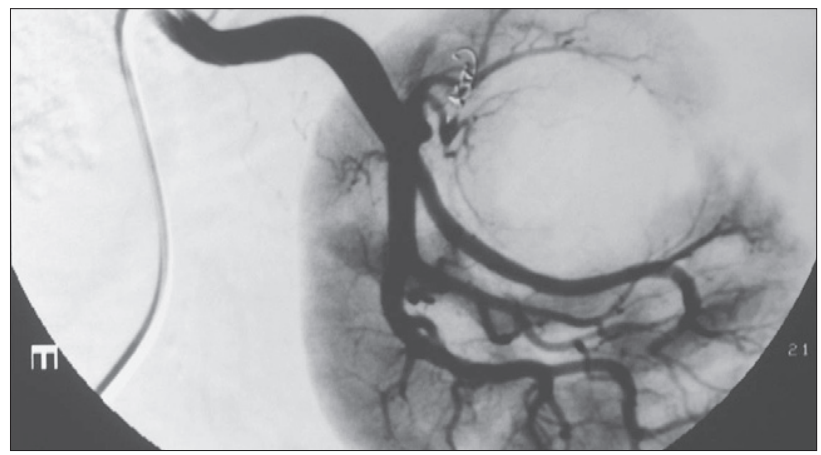

Fig. 4. Renoangiography: Afferent arteriole of pseudoaneurysm obturated by an endovascular body (see Fig. 1).

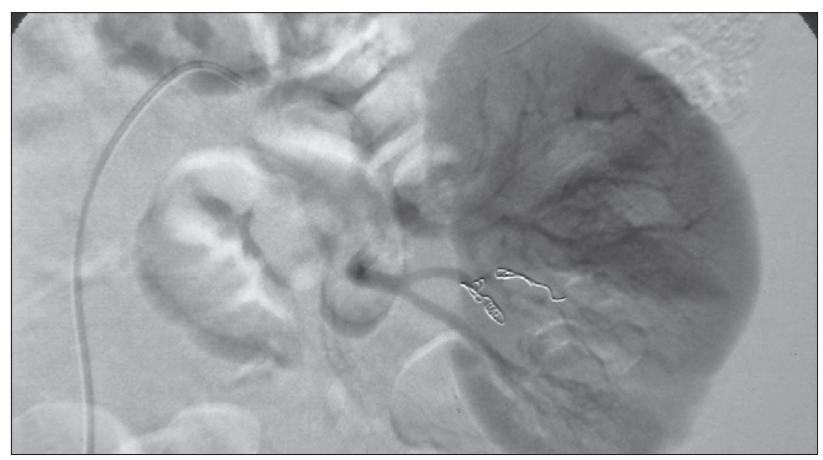

Fig. 5. Renoangiography: Afferent arterioles of two pseudoaneurysms obturated by two endovascular bodies (see Fig. 2).

stration of a congenital anomaly of renal blood vessels (Currie et al, 1964). The appearance of arteriovenous fistulae may be accompanied by the formation of hollow pseudoaneurysms, particularly in cases when surgery distorts the integrity and compactness of renal parenchyma, thus allowing extravasation of blood into immediate vicinity of the fistula. As long as the arteriovenous fistula is caused by needle renal biopsy, pseudoaneurysm does not usually develop. In such a case, the arteriovenous fistula manifests dominantly as a hyperkinetic blood circulation that particularly in older patients may eventually lead to cardiac failure (Maldonado et al, 1964, Voiculescu et al, 2003). Haemodynamic alterations induced by arteriovenous fistula in the kidney include ischaemia of renal parenchyma distally to the fistula with subsequent diastolic hypertension. Haemodynamically insignificant arteriovenous fistulae induced by renal biopsy are usually asymptomatic and a part of them heal spontaneously by local thrombosis (Riehl et al, 2005, Ibanez-Pradas et al, 2006).

The arteriovenous fistula may give rise to pseudoaneurysms varying in size, number and shape, even with multiple voids as observed in our own clinical material. Fistulae may communicate with the collecting system of the kidney and manifest themselves in massive, often life-threatening haematuria (Riehl et al, 2005). When resecting renal carcinomas extending to the central complex of the kidney, namely the calix is often opened. Less frequently, the fistulae manifest themselves in mild intermittent haematuria or slight blunt pain in the operated kidney which sometimes cannot be easily distinguished from the pain caused by operation.

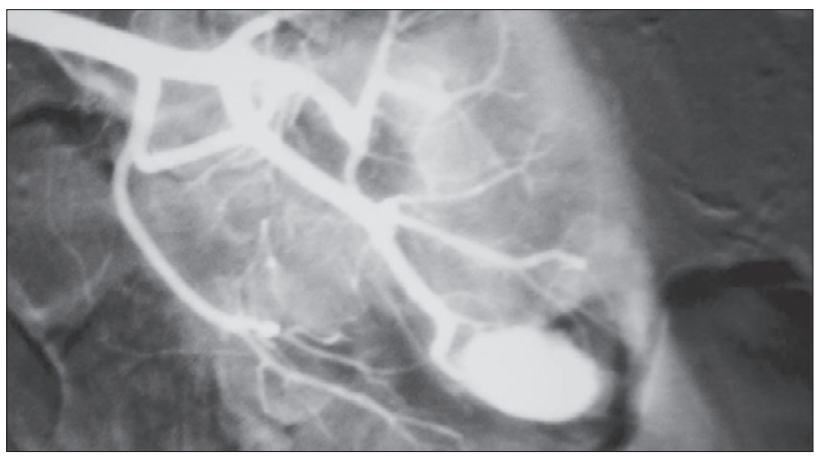

Fig. 6. Renoangiography: Arterial phase - arterio-venous fistula with pseudoaneurysm.

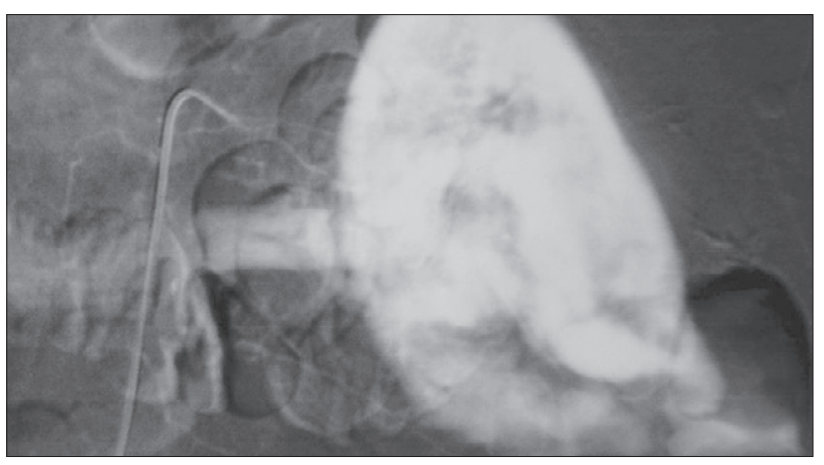

Fig. 7. Renoangiography: Venous phase - fast leakage of contrast medium via renal vein.

The diagnosis of a symptomatic arteriovenous fistula must be fast and precise. On physical examination, one can hear murmur above the affected kidney, which is a manifestation of turbulent blood flow in the fistula or pseudoaneurysm. This phenomenon is much easier to perceive above a transplanted kidney right below the abdominal wall (Voiculescu et al, 2003, Barley et al, 2006). On ultrasonographic examination of renal parenchyma one can see a hypoechogenous to anechogenous formation varying in size. In this formation, the turbulent blood flow can be proved by Doppler flowmetry characterised by a typical signal with changed flow waves (Subramanyam et al, 1983, Gainza et al, 1995, Carrieri et al, 1996, Kabaalioglu et al, 1997).

Precise examinations include CT or angioCT and MR by which one can accurately determine the place, size and shape of pseudoaneurysm and prove the arteriovenous fistula (Selli et al, 1986, Primus et al, 2007).

In our experience, digital subtraction renoangiography and direct catheterisation of renal artery by Seldinger's method not only has the highest diagnostic efficacy but it is also the first step in active treatment by selective embolisation of the arteriovenous fistula and/or by inserting endovascular coils (Lopatkin and Mazo, 1969, Swana et al, 1996, Dorffner et al, 1998, Barley et al, 2006). In angiography, a sudden, as if abruptly cut end of the intrarenal artery is shown with direct visualization of the void of pseudoaneurysm, and filling of the renal veins. Sometimes dilation of a branch of renal artery may be found supplying the arteriovenous fistula and immediately on applying a contrast substance also the dilated 
renal vein is visualized. The shown pathomorphologic pattern has to be evaluated in detail, it is necessary to apply embolisation material of a diameter exceeding the lumen of the afferent artery so as to prevent potential pulmonary embolisation.

For embolisation we use the $5 \mathrm{~F}$ guiding catheter and micro instruments ( 0.021 inch microcatheter, 0.018 inch microspirals of complex shape usually 3 or $4 \mathrm{~mm}$ in diameter, and a pusher). We have good experience with the method of endovascular superselective embolisation. This method allows preserving the altered kidney, virtually without damaging other preserved intrarenal arteries, thus without damaging the renal parenchyma.

As far as the open surgical methods of treating the arteriovenous fistulae are concerned, they can be methodologically classified as operations in vivo and ex vivo (Lang a Schott, 2005). The essence of all methods lies in insulated ligation of the segmental branch of renal artery supplying the arteriovenous fistula or resection of a part of the kidney with the arteriovenous fistula. In literature, cases are described in which arteriovenous fistulae after needle renal biopsy were treated in this way. The open surgical revision and reconstruction of a previously operated kidney is surely difficult enough and complicated. We do not have experience in this field.

In case of massive haematuria with a life-threatening haemorrhagic shock, a prompt surgical revision and nephrectomy of the inflicted kidney is the method of choice. In this way we resolved bleeding in two patients. Peroperatively, we found a markedly dilated renal artery and vein in a way discussed above.

In the context of treatment of arteriovenous fistulae with pseudoaneurysms of renal artery developed as a complication after extirpation of a localised renal carcinoma, in the first place one has to emphasise the consequential prevention of its formation. Nevertheless, not even careful haemostasis by suture ligatures of all the bleeding places on the sectional wall after resecting a part of the kidney inflicted by the tumour is a sufficient guarantee for avoiding the formation of arteriovenous fistula. When resecting the kidney, all bleeding places must be consequently treated by sutures. Smaller parenchymal bleeding may be stopped by using tissue glue. Afterwards, when reconfiguring the kidney, it is appropriate to insert thromboplastic material (synthetic or autologous) into the void after tumour resection, and the opposite walls of the defect should be sutured in one or two layers by mattress stitches so that no free space remains between them.

\section{Conclusion}

Arteriovenous fistula with pseudoaneurysm of the renal artery is a rare but potentially severe or even life-threatening complication occurring after various invasive renal interventions. One of the causes giving rise to a fistula between close-placed branches of renal arteria and vein is the damage incurred to these vessels when resecting the localised renal tumour.

The symptomatic arteriovenous fistula requires fast and precise diagnosis enabling effective treatment. Endovascular superselective coil embolisation is a successful method allowing the preservation of the kidney inflicted by arteriovenous fistula with pseudoaneurysm of renal artery.

\section{References}

1. Baláž V, Babel'a J, Okapec S. Renal artery pseudoaneurysm following partial nephrectomy for RCC. Eur Urol Suppl 2007; 6: 235.

2. Barley FL, Kessel D, Nicholson T, Robertson I. Selective embolization of large symptomatic iatrogenic renal transplant arteriovenous fistula. Cardiovasc Intervent Radiol 2006; 29: 1084-1087.

3. Breza J Jr. Pseudoaneuryzma arteria renalis po resekcii obličky. In: Breza J, Marenčák J, Minčík I et al. Nádory obličiek. Bratislava: OG -Vydavatel'stvo Pol'ana, s.r.o., 2008, 368 p.

4. Carrieri G, Battaglia M, Martino P, Ditonno P, Tempesta A, Selvaggi FP. Role of color Doppler echography (ECD) in the diagnosis and follow-up of post-biopsy arteriovenous fistula in the transplanted kidney. Arch Ital Urol Androl 1996; 68 (Suppl 5): 87-89.

5. Currie JCM, Thomas ML, Pinninger JL. Congenital intrarenal arteriovenous fistula. Br J Surg 1964; 51: 40-44.

6. Dorffner R, Thurnher S, Prokesch R, Baniker A, Turetschek K, Schmidt A, Lammer J. Embolization of iatrogenic vascular injuries of renal transplants: Immediate and follow-up results. Cardiovasc Intervent Radiol 1998; 21: 129-134.

7. Gainza FJ, Minguela I, Lopez-Vidaur I, Ruiz LM, Lampreabe J. Evaluation of complications due to percutaneous renal biopsy in allografts and native kidneys with color-coded Doppler sonography. Clin Nephrol 1995; 43: 303-308.

8. Ibanez Pradas V, Garcia Vila JH, Redondo Ibanez M, Diaz Ramon C. Spontaneous resolution of intrarenal pseudoaneurysm. Eur J Pediat Surg 2006; 29: 362-364.

9. Kabaalioglu A, Yilmaz S, Sindel T, Ozkaynak C, Luleci E. Renal vein aneurysm: Diagnosis with color Doppler sonography. Amer J Radiol 1997; 168: 645-646.

10. Kaufman JJ, Gordon A, Maxwell MH. Intrarenal arteriovenous fistula following needle biopsy of the kidney. Calif Med 1965; 103: 350-354.

11. Lang W, Schott GE. Treatment of multiple renal artery aneurysms - A case report to demonstrate treatment options. Zbl Chir 2005; 130: 585-588.

12. Lopatkin NA, Mazo EB. Aneurysm of the renal artery. Int Urol Nephrol 1969; 1: 141-152.

13. Maldonado JE, Sheps SG, Bernatz PE, DeWeerd JH, Harrison EG Jr. Renal arteriovenous fistula: A reversible cause of hypertension and heart failure. Amer J Med 1964; 37: 499-513.

14. Primus H, Samtleben M, Schmitt E. Renale arteriovenose Fistel. Urologe A 2007; 46: 412-415.

15. Riehl J, Maigatter S, Schacht B, Kierdorf H, Sieberth HG. Arteriovenous fistula and bleeding into the renal pelvis after percutaneous renal biopsy. Ultraschall Med 1993; 14: 285-289.

16. Selli C, Bartolozzi C, Lizzadro G, Petacchi D. Arteriovenous fistula associated with renal cell carcinoma: Demonstration by magnetic resonance imaging. Urol Radiol 1986; 8: 190-193.

17. Sheps SG, Maldonado JE. Arteriovenous renal fistula. Review of 109 cases. Klin Wschr 1969; 47: 621-628.

18. Subramanyam BR, Lefleur RS, Bosniak MA. Renal arteriovenous fistula and aneurysm: Sonographic findings. Radiology 1983; 149: 261-263.

19. Swana HS, Cohn SM, Burns GA, Egglin TK. Renal artery pseudoaneurysm after blunt abdominal trauma: Case report and literature review. J TraumaInjury Infect Crit Care 1996; 40: 459-461.

20. Voiculescu A, Brause M, Engelbrecht V, Sandmann W, Pfeiffer T, Grabensee B. Hemodynamically relevant hematuria several months after biopsy of a kidney graft: An unusual cause. Clin Nephrol 2003; 59: 217-221.

21. Zumtobel V. Arterio-venose Fisteln nach Massenligaturen. Langenbecks Arch Surg 1973; 334: 925-926.

Received September 8, 2010. Accepted February 14, 2012. 